\section{Improving Care for All Pediatric Patients With Hodgkin Lymphoma}

Jamie E. Flerlage, MD, MS

T he curability of Hodgkin lymphoma $(\mathrm{HL})$ has remained above $90 \%$ for decades, but exactly "how" we cure our patients is critically important to minimize late effects of therapy for survivors. From the earliest cure that used radiotherapy alone in the early 20th century to the newest drug on the market today, every treatment has risks and benefits. As the field of medicine constantly evolves before us, our job as oncologists is to learn from each patient we treat and strive to cure tomorrow's patients better than we can today.

Sadly, survivors of $\mathrm{HL}$ have demonstrated the natural progression of long-term treatment toxicities from combined-modality therapy with chemotherapy and radiation. Secondary malignancies, cardiopulmonary toxicity, infertility, and endocrine dysfunction have affected countless survivors. For years we attributed the 17-fold increased risk for the development of breast cancer in long-term survivors solely to the use of high radiation doses and large fields. ${ }^{1}$

However, as sequential trials reduced radiation dose, field size, and the number of patients and sites receiving radiation, we now see other factors associated with an increased risk for developing breast cancer, such as a cumulative dose of doxorubicin $>250 \mathrm{mg} / \mathrm{m}^{2}$. ${ }^{2}$ Although late effects could be considered a "luxury" of survivors, rigorous survivorship research has informed adaptations to treatment regimens that allow $\mathrm{HL}$ survivors today to have a lower risk profile than those a decade before.

For pediatric $\mathrm{HL}$, iterations of clinical trials performed by large consortia use outcome and toxicity data from previous protocols to inform development of the subsequent trial. This has succeeded in both maintaining high cure rates and minimizing overall treatment intensity through the eras.

At the same time, because the outcomes of most trials have an overall survival rate of $90 \%$ to $95 \%$, providers face the new challenge of helping patients choose among several regimens with similar survival. Although the standard of care will always remain enrollment on an open clinical trial, the lack of standardized, evidence-based guidelines has led to significant variation in treatment practices for patients treated off-protocol. Therefore, the formation of NCCN Clinical Practice Guidelines in Oncology (NCCN Guidelines) for Pediatric HL (included in this issue) ${ }^{3}$ provide useful guidance. These guidelines provide a framework not only for treatment decisions but also for the entire process, from initial workup and staging through follow-up and relapse.

Staging and response assessment for pediatric HL are nuanced and challenging given the rarity of this disease, and therefore the relative infrequency with which they are performed at any institution. The NCCN Guidelines provide up-to-date recommendations that may differ from current institutional practice patterns. Examples of this include the omission of both bone marrow biopsies for the staging of $\mathrm{HL}$ and of surveillance imaging for any patient after the end of therapy unless there is a clinical concern for relapse.

See page 733 for related article.

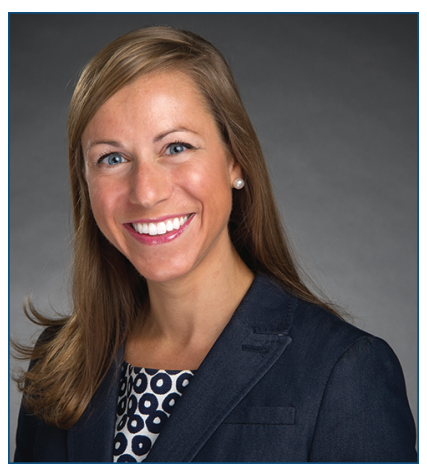

JAMIE E. FLERLAGE, MD, MS

Jamie E. Flerlage, MD, MS, is an Assistant Member in the Department of Oncology at St. Jude Children's Research Hospital, leads the St. Jude-Stanford-Dana-Farber Pediatric Hodgkin Consortium, and serves as PI of the current frontline trial. She obtained her undergraduate and medical degrees at Union College and Albany Medical College in Albany, New York, and completed pediatric residency at Johns Hopkins Children's Center. Dr. Flerlage performed pediatric hematology/ oncology training at St. Jude Children's Research Hospital. In an effort to improve the care for pediatric Hodgkin lymphoma around the world, she leads an

international working group, SEARCH for CAYAHL, to harmonize staging and response criteria across all consortia. She serves as a member of the Children's Oncology Group Hodgkin Lymphoma steering committee, is a co-leader of NODAL, the Hodgkin subgroup of the Pediatric Cancer Data Commons, and most recently co-founded a new pediatric and adult global initiative with Michael Binkley from Stanford called GLOW (Global nLPHL One Working group).

doi: 10.6004/jnccn.2021.7064

The ideas and viewpoints expressed in this commentary are those of the author and do not necessarily represent any policy, position, or program of NCCN. 
Of note, this early version of the guidelines focuses on classical $\mathrm{HL}$ and does not include treatment algorithms for patients with nodular lymphocyte-predominant $\mathrm{HL}$, because not enough evidence is available to support one treatment over another for this biologically distinct disease. As evidence emerges, the guidelines will be expanded to include treatment strategies for nodular lymphocyte-predominant HL.

These guidelines have never been more important than they are now, as COVID-19 has challenged the world's ability to travel, including for medical care and enrollment on clinical trials. This has left many providers faced with treating and managing these young patients at local institutions. I believe these NCCN Guidelines help promote equality and ensure optimal care for all patients with $\mathrm{HL}$.

Disclosures: Dr. Flerlage has disclosed having no financial interests, arrangements, or affiliations with the manufacturers of any products discussed in this article or their competitors.

Correspondence: Jamie E. Flerlage, MD, MS, St. Jude Children's Research Hospital, 262 Danny Thomas Place, MS 260, Memphis, TN 38105. Email: jamie.flerlage@stjude.org

\section{References}

1. Castellino SM, Geiger AM, Mertens AC, et al. Morbidity and mortality in long-term survivors of Hodgkin lymphoma: a report from the Childhood Cancer Survivor Study. Blood 2011;117:1806-1816.

2. Ehrhardt MJ, Howell CR, Hale K, et al. Subsequent breast cancer in female childhood cancer survivors in the St Jude

Lifetime Cohort Study (SJLIFE). J Clin Oncol 2019;37: 1647-1656.

3. Flerlage JE, Hiniker SM, Armenian S, et al. NCCN Clinical Practice Guidelines in Oncology for Pediatric Hodgkin Lymphoma, Version 3.2021. J Natl Compr Canc Netw 2021;19:733-754.

\section{Advances in Multidisciplinary Management of Acute Myeloid Leukemia}

Thursday, June 17, 2021 12:00 - 1:00 PM EDT

Daniel A. Pollyea, MD, MS

Glen J. Peterson, DNP, ACNP, RN Jennifer Tobin, PharmD, DPh University of Colorado Cancer Center

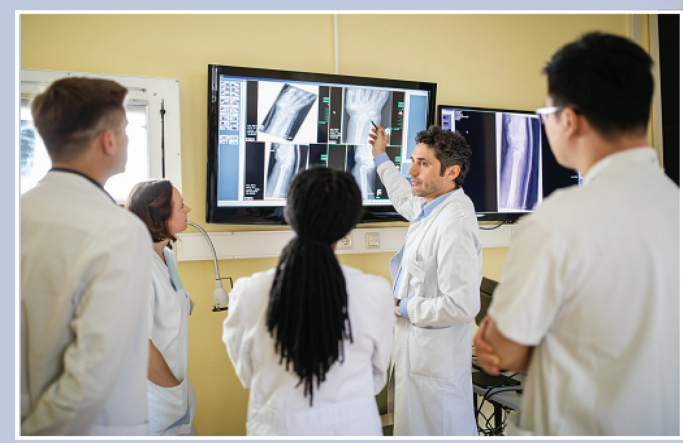

Visit education.nccn.org/tumorboards to register for upcoming webinars or to view recorded presentations.

This activity is supported by educational grants from AstraZeneca, Blueprint Medicines, Epizyme Inc., GlaxoSmithKline, Lilly, Novartis, Puma Biotechnology, and Sanofi Genzyme. This activity is supported by independent medical education grants from Bristol Myers Squibb, Illumina, Inc, and Pharmacyclics LLC, an AbbVie company and Janssen Biotech, Inc. This activity is supported by an independent educational grant from Astellas. This educational activity is supported by a medical education grant from Exelixis, Inc. 